See discussions, stats, and author profiles for this publication at: https://www.researchgate.net/publication/348663766

\title{
BELAJAR MUDAH KIMIA KARBON
}

Chapter · January 2010

1 author:

(2) Syamsul Bakhri

Universitas Muslim Indonesia

5 PUBLICATIONS 0 CITATIONS

SEE PROFILE 


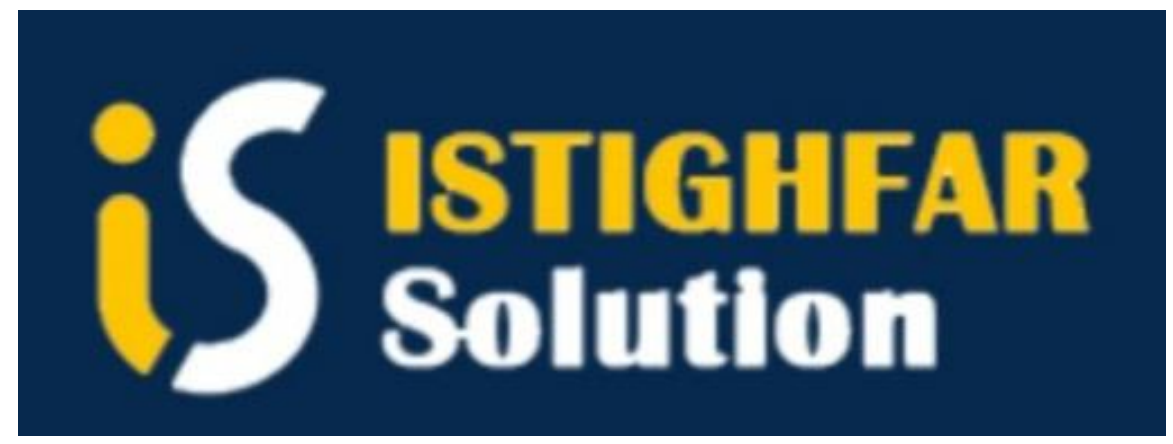

BISMILLAAHIRRAHMAANIRRAHIIM

Assalaamu 'Alaikum Warahmatullaahi Wabarakaatuh,

Puji Syukur hanya dipanjatkan kepada ALLAH SWT, serta Salawat dan Salam kepada Nabi kita Rasulullah Muhammad Sallallaahu 'Alaihi Wassallam.

Makalah ini, merupakan Kesimpulan atau Garis - garis Besar tentang Kimia Karbon, yang menitik-beratkan pada Reaksi - reaksi yang terjadi pada Kimia Karbon beserta Sifat sifatnya.

Paper atau Makalah ini, dibuat agar siswa SMA dapat lebih Mudah Memahami tentang KIMIA KARBON.

Makalah ini, Saya persembahkan kepada Anak - anakku Siswa Kelas XI dan XII IPA, khusunya kepada Anak - anakku Peserta Bimbingan Khusus di ISTIGHFAR SOLUTION, guna menghadapi Ujian Nasional dan SBMPTN, sehingga diharapkan dapat Menjawab berbagai bentuk (macam) soal tentang Kimia Karbon pada Ujian Nasional dan SBMPTN.

Semoga Makalah ini, Bermanfaat bagi Anak - anakku Siswa Kelas XI dan XII IPA, khususnya Peserta Bimbingan Khusus di ISTIGHFAR SOLUTION.

Semoga Allaah Swt, Senantiasa Melimpahkan Rahmat, Karunia, dan Hidayah - NYA kepada kita semua... Aaminn Yaa Rabbal 'Aalamiin.

Wassalam,

Penulis, 


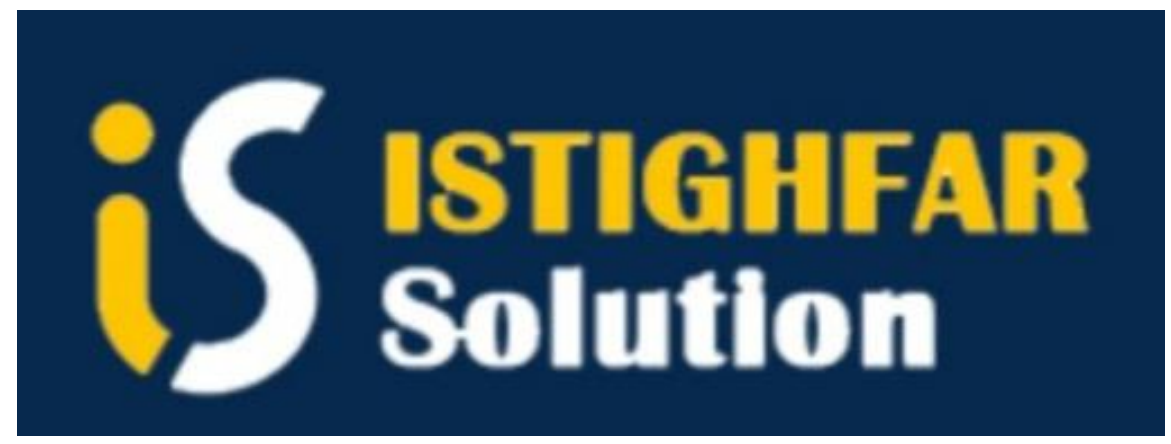

\section{BELAJAR MUDAH}

\section{KI M I A K A R B O N}

\section{REAKSI - REAKSI KIMIA KARBON}

\section{A. REAKSI SUBSTITUSI}

Merupakan reaksi dimana terjadi PENUKARAN / PENGGANTIAN suatu atom atau gugus atom dari suatu seyawa karbon oleh atom atau gugus atom dari senyawa lain.

$$
R-X+R^{\prime}-Y \rightarrow R-Y+R^{\prime}-X
$$

1. REAKSI HIDROLISIS

$$
\begin{aligned}
& >\quad \text { ALKIL HALIDA }+ \text { AIR } \rightarrow \text { ALKOHOL + ASAM KUAT (ASAM HALIDA) } \\
& >\quad \text { ESTER + AIR } \rightarrow \text { ASAM KARBOKSILAT + ALKOHL }
\end{aligned}
$$

2. REAKSI ESTERIFIKASI

$$
\text { ASAM KARBOKSILAT + ALKOHOL } \rightarrow \text { ESTER + AIR }
$$




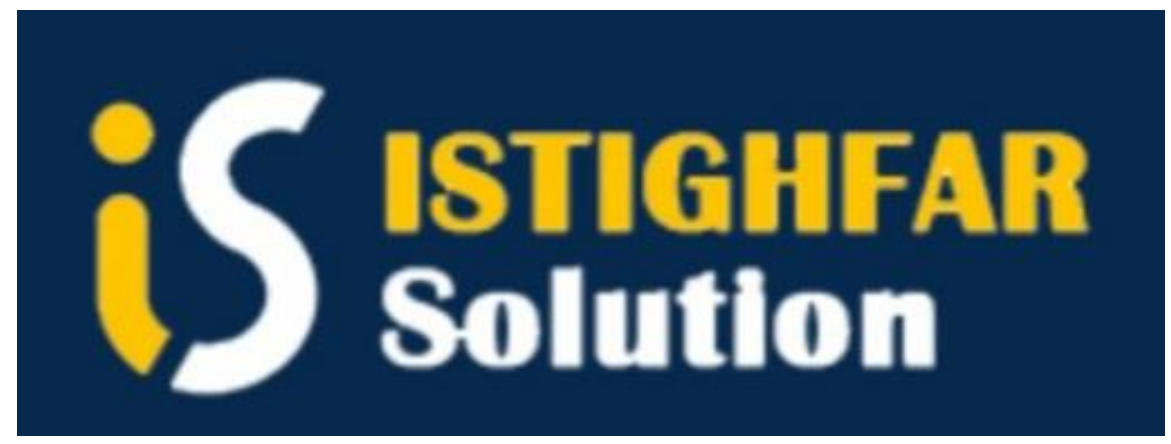

3. REAKSI SAPONIFIKASI (PENYABUNAN)

$$
\begin{aligned}
& >\text { LEMAK + BASA KUAT } \rightarrow \text { SABUN + GLISEROL } \\
& >\text { ESTER + BASA KUAT } \rightarrow \text { SABUN + ALKOHOL }
\end{aligned}
$$

4. SINTESA WURTZ

$$
\begin{aligned}
& \text { ALKIL HALIDA + } 2 \mathrm{Na}+\text { ALKIL HALIDA } \rightarrow \text { ALKIL - ALKIL + GARAM KUAT } \\
& \mathrm{R}-\mathrm{X}+2 \mathrm{Na}+\mathrm{R}^{\prime}-\mathrm{X} \rightarrow \mathrm{R}-\mathrm{R}^{\prime}+2 \mathrm{NA}-\mathrm{X}
\end{aligned}
$$

5. REAKSI KHLORINASI

$$
\begin{array}{llll}
\mathrm{R}-\mathrm{H}+\mathrm{X}-\mathrm{X} & \stackrel{\text { S.U.V }}{\longrightarrow} \mathrm{R}-\mathrm{X}+\mathrm{H}-\mathrm{X} \\
>\quad \mathrm{CH}_{4} & +\mathrm{Cl}-\mathrm{Cl} \stackrel{\text { s.U.V }}{\longrightarrow} \mathrm{CH}_{3}-\mathrm{Cl}+\mathrm{H}-\mathrm{Cl} \\
>\quad \mathrm{CH}_{3}-\mathrm{Cl}+\mathrm{Cl}-\mathrm{Cl} \stackrel{\text { S.U.V }}{\longrightarrow} \mathrm{CH}_{2}-\mathrm{Cl}_{2}+\mathrm{H}-\mathrm{Cl} \\
>\quad \mathrm{CH}_{2}-\mathrm{Cl}_{2}+\mathrm{Cl}-\mathrm{Cl} \stackrel{\text { s.U.V }}{\longrightarrow} \mathrm{CH}-\mathrm{Cl}_{3}+\mathrm{H}-\mathrm{Cl} \\
>\quad \mathrm{CH}-\mathrm{Cl}_{3}+\mathrm{Cl}-\mathrm{Cl} \stackrel{\text { s.U.V }}{\longrightarrow} \mathrm{C}-\mathrm{Cl}_{4} \text { (Khlorinasi) }+\mathrm{H}-\mathrm{Cl}
\end{array}
$$

6. REAKSI ALKIL HALIDA + BASA KUAT

$$
\mathrm{R}-\mathrm{X}+\mathrm{NaOH} \rightarrow \text { ALKOHOL + GARAM KUAT }
$$




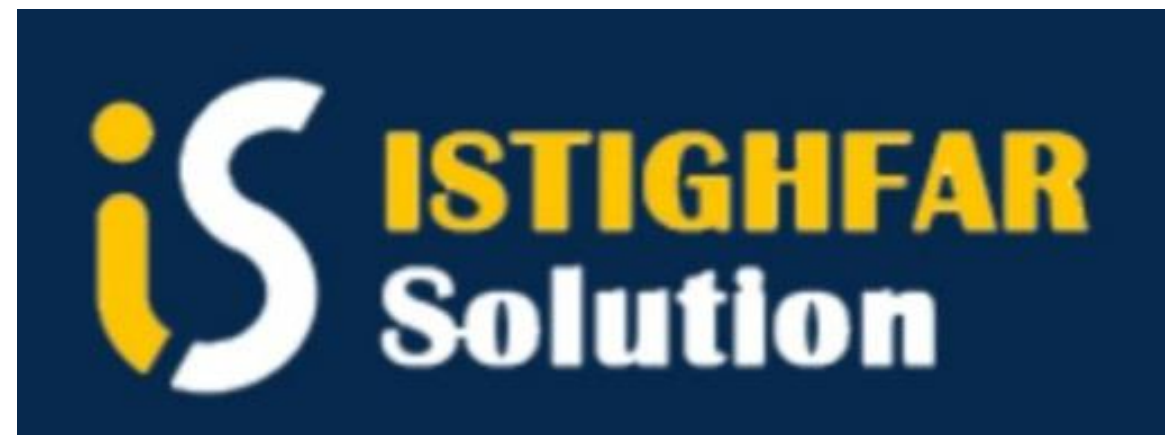

7. REAKSI ALKOHOL Dengan $\mathrm{PCl}_{3}$

ALKOHOL $+\mathrm{PCl}_{3} \rightarrow$ ALKIL HALIDA $+\mathrm{H}_{3} \mathrm{PO}_{3}$

8. REAKSI ALKOHOL Dengan LOGAM Na

$\mathrm{ALKOHOL}+\mathrm{Na} \rightarrow \mathrm{R}-\mathrm{ONa}+\mathrm{H}_{2}$

KETERANGAN,

Reaksi 7 dan 8, digunakan untuk membedakan ALKOHOL dan ETER (Mempunyai Rumus Molekul yang sama, dengan gugus fungsi yang berbeda.

$$
\begin{array}{lll}
>\text { REAKSI 7, } & \mathrm{R}-\mathrm{OH}+\mathrm{PCl}_{3} \rightarrow \mathrm{H}_{3} \mathrm{PO}_{3} \\
& \mathrm{R}-\mathrm{O}-\mathrm{R}^{\prime}+\mathrm{PCl}_{3} \quad \text { TIDAK BEREAKSI } \\
& \\
& \mathrm{R}-\mathrm{OH}+\mathrm{NA} \rightarrow \text { Menghasilkan Gas } \mathrm{H}_{2} \\
\mathrm{R}-\mathrm{O}-\mathrm{R}^{\prime}+\mathrm{PCl}_{3} \quad \text { TIDAK BEREAKSI }
\end{array}
$$




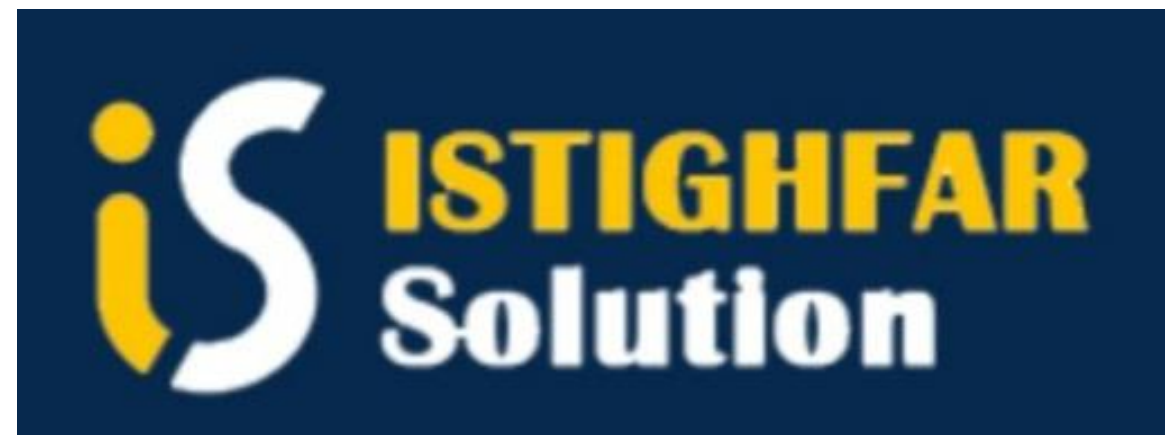

\section{B. REAKSI ADISI}

Reaksi dimana terjadi PENANGKAPAN / PERUBAHAN atom-atom lain, sehingga terjadi PENGUBAHAN dari ikatan tidak jenuh menjadi Ikatan jenuh.

Reaksi Adisi, hanya dapat dialami oleh zat-zat yang mengandung Ikatan Tak Jenuh atau Ikatan Rangkap.

1. REAKSI HIDROGENASI (ADISI Dengan $\mathrm{H}_{2}$ )

$>$ REAKSI HIDROGENASI ALKENA

ALKENA $+\mathrm{H}_{2} \rightarrow$ ALKANA

REAKSI HIDROGENASI ALDEHID

ALDEHID + H2 $\rightarrow$ ALKOHOL

2. REAKSI ADISI Dengan HALOGEN

ALKENA + HALOGEN $\rightarrow$ HALOGEN ALKANA

Gunananya, Untuk membedakan senyawa ALKENA dengan SIKLOAKKANA, karena kedua senyawa mempunyai isomer fungsional (Rumus Molekul sama, tetapi Gugus fungsi berbeda).

Nah, hasil pengamatannya, memanfaatkan warna dari Brom yaotu MERAH COKLAT berubah menjadi BENING. Sedangkan SIKLOALKANA tidak bereaksi dengan Brom. 


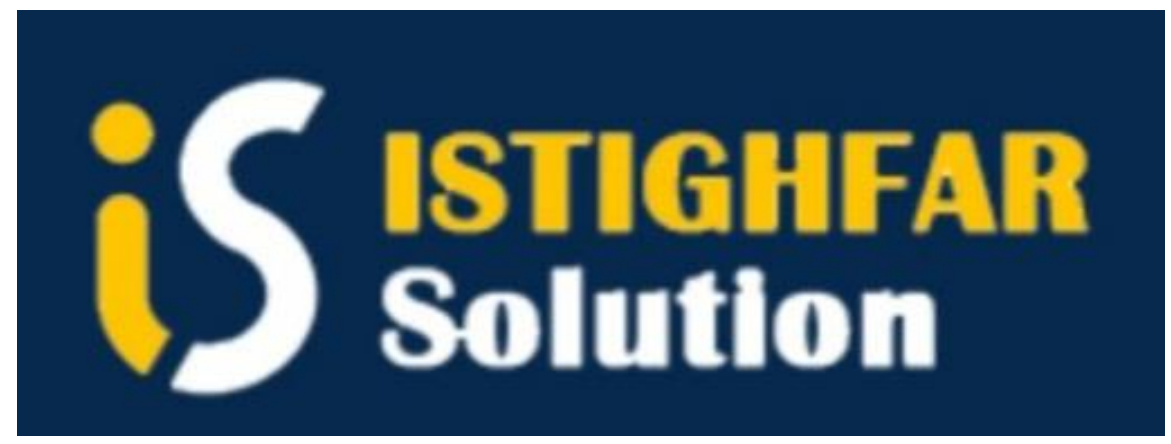

3. REAKSI ADISI Dengan ASAM HALIDA (HX) / ADISI MARKOVNIKOV

$\checkmark \quad$ ALKENA + HX $\rightarrow$ HALIDA ALKANA

$\mathrm{R}-\mathrm{CH}=\mathrm{CH}-\mathrm{R}^{\prime}+\mathrm{HX} \rightarrow \mathrm{R}-\mathrm{CH} 2-\mathrm{CHX}-\mathrm{R}^{\prime}$

$\checkmark \quad$ Ikatan Rangkap merupakan kumpulan electron

$\checkmark \quad$ Gugus Alkil, merupakan GUGUS PENDORONG ELEKTRON

Jika ALKIL makin Besar ( ALKIL 》), Maka Daya Dorong Makin Kuat.

$\ldots-\mathrm{CH}_{3}<\ldots-\mathrm{C}_{2} \mathrm{H}_{5}<\ldots-\mathrm{C}_{3} \mathrm{H}_{7}$ 


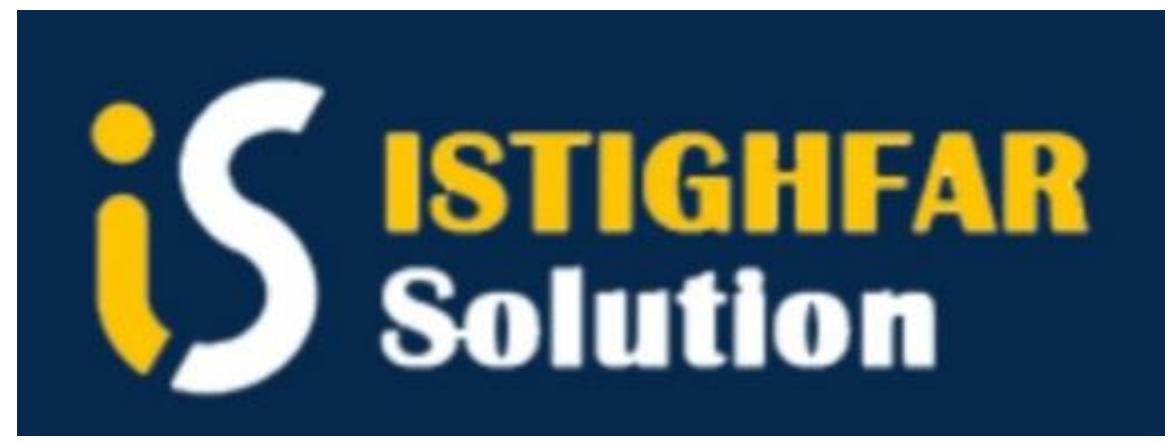

\section{REAKSI ELIMINASI}

Merupakan reaksi PENGHILANGAN (PENARIKAN) beberapa atom atau gugus atom, untuk mendapatkan zat baru.

Reaksi ini merupakan kebalikan dari Reaksi Adisi.

$$
\mathrm{CX}-\mathrm{CY} \rightarrow \mathrm{C}=\mathrm{C}+\mathrm{X}-\mathrm{Y}
$$

1. PENARIKAN $\mathrm{H}_{2} \mathrm{O}$ (DEHIDRASI)

$$
\begin{aligned}
& >\quad \text { ALKOHOL } \stackrel{-H 2 O \text { dengan bantuan } \mathrm{H} 2 S O 4(P)}{\longrightarrow} \text { ALKENA }\left(170^{\circ} \mathrm{C}-180^{\circ} \mathrm{C}\right) \\
& >\quad \mathrm{ALKOHOL}+\mathrm{ALKOHOL} \stackrel{-H 2 O \text { dengan bantuan H2SO4 }(P)}{\longrightarrow} \text { ETER }\left(140^{\circ} \mathrm{C}\right)
\end{aligned}
$$

2. PENARIKAN HCl

$$
\mathrm{R}-\mathrm{OH}-\mathrm{Cl} \stackrel{-\mathrm{HCl} \text { dengan bantuan } \mathrm{KOH} \text { Alkoholik }}{\longrightarrow} \text { ALKENA }
$$

\section{DEHIDROGENASI (PELEPASAN $\mathrm{H}_{2}$ )}

$$
\mathrm{R}-\mathrm{CH}-\mathrm{H}-\mathrm{CH} \mathrm{H}-\mathrm{R}^{\prime} \stackrel{\text { KaTALIS }(\mathrm{Ni}) \text { dengan bantuan Pemanasan }}{\longrightarrow} \mathrm{R}-\mathrm{CH}=\mathrm{CH}-\mathrm{R}^{\prime}+\mathrm{H}_{2}
$$

4. DEHIDROHALOGENASI

$$
\mathrm{R}-\mathrm{CH}-\mathrm{H}-\mathrm{CHX}-\mathrm{R}^{\prime} \stackrel{\text { BASA }(\mathrm{NaOH}) \text { dengan bantuan Pemanasan }}{\longrightarrow} \mathrm{R}-\mathrm{CH}=\mathrm{CH}-\mathrm{R}^{\prime}+\mathrm{HX}
$$




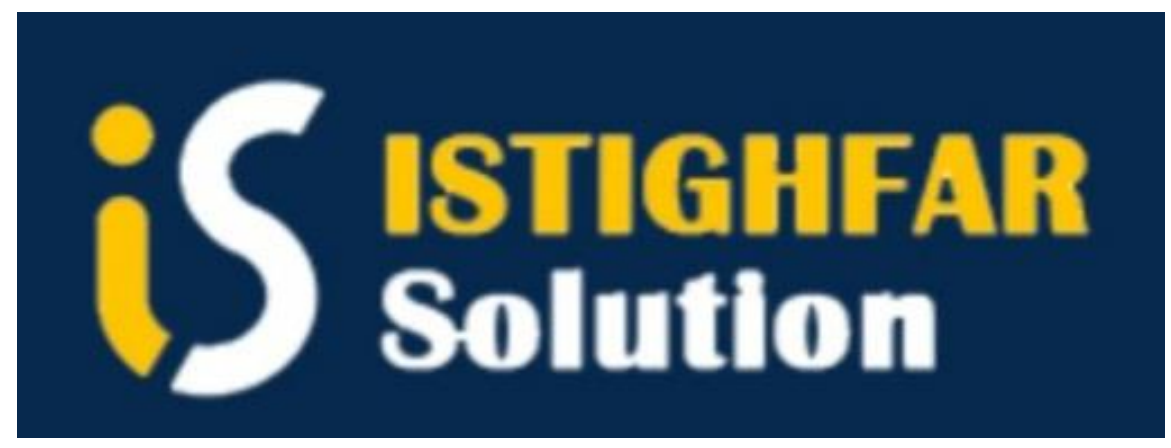

D. REAKSI REDOKS / OKSIDASI

Sifat Reduktor, atau yang dapat mengalami Oksidasi.

1. OKSIDASI Dengan Larutan $\mathrm{KMnO}_{4} / \mathrm{K}_{2} \mathrm{Cr}_{2} \mathrm{O}_{7}$

ALKOHOL $\begin{cases}\{\text { PRIMER } \stackrel{o}{\rightarrow} \text { ALDEHID } & \stackrel{o}{\rightarrow} \text { ASAM KARBOKSILAT } \\ & \text { SEKUDER } \stackrel{o}{\rightarrow} \text { KETON }\end{cases}$

2. OKSIDASI Dengan OZON (OZONALISA)

ALKENA + $03 \rightarrow$ ASAM KARBOKSILAT + ALKANON

3. OKSIDASI Dengan Larutan Fehling dan Benedict

ALDEHID + CuO (Fehling) $\rightarrow$ ASAM KARBOKSILAT $+\mathrm{Cu}_{2} \mathrm{O}$ (Merah) 


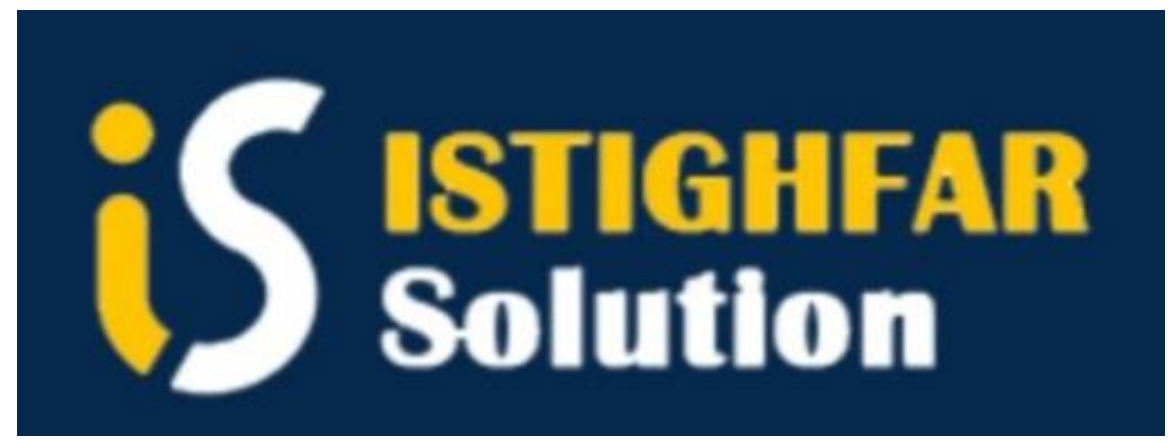

II. ISOMER GUGUS FUNGSI

1. ALKOHOL

ALKOHOL (DAPAT DIOKSIDASI)

$$
\text { ETER (TIDAK) }
$$

ALDEHID (DAPAT BEREKSI DENGAN FEHLING)

2. ALDEHID

$$
\text { KETON (TIDAK) }
$$

ASAM KARBOKSILAT (MEMERAHKAN LAKMUS BIRU)

3. ASAM KARBOKSILAT

$$
\text { ESTER (HARUM \& DAPAT DIHIDROLISIS) }
$$

4. ALKENA

$$
\text { ALKENA (DAPAT DIADISI) }
$$

SIKLOALKANA (TIDAK) 


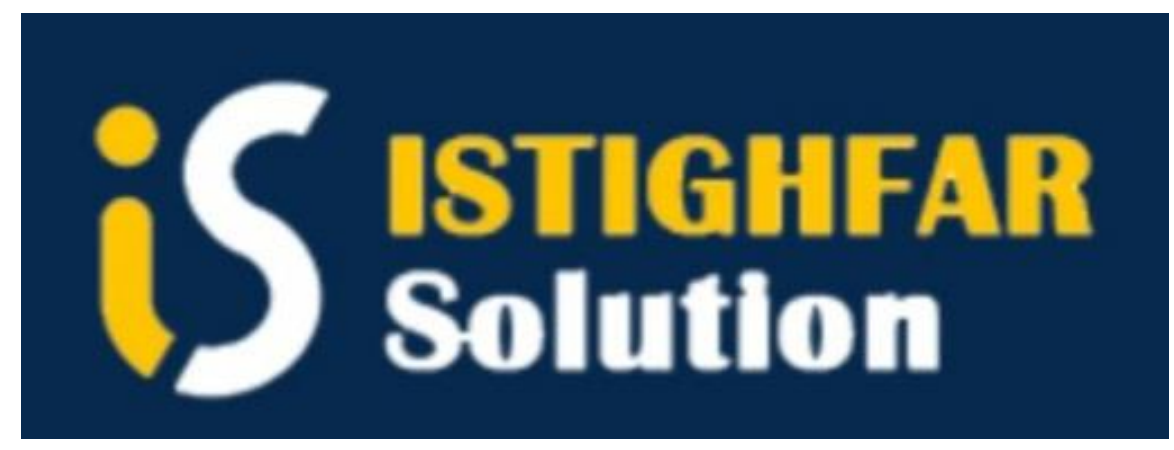

III. ISOMER GEOMETRIS

Ciri - cirinya,

$\checkmark \quad$ Ada ikatan rangkap dua (ALKENA)

$\checkmark \quad$ Pada C rangkap HARUS mengikat dua gugus lain yang berbeda.

1. ISOMER CIS

$\mathbf{R}$

$C====C$

B

B

2. ISOMER TRANS

$\mathbf{R}$

B

$C====C$

B

B
$\mathbf{R}=\mathbf{M r}$ Kecil

$B=$ Mr Besar 


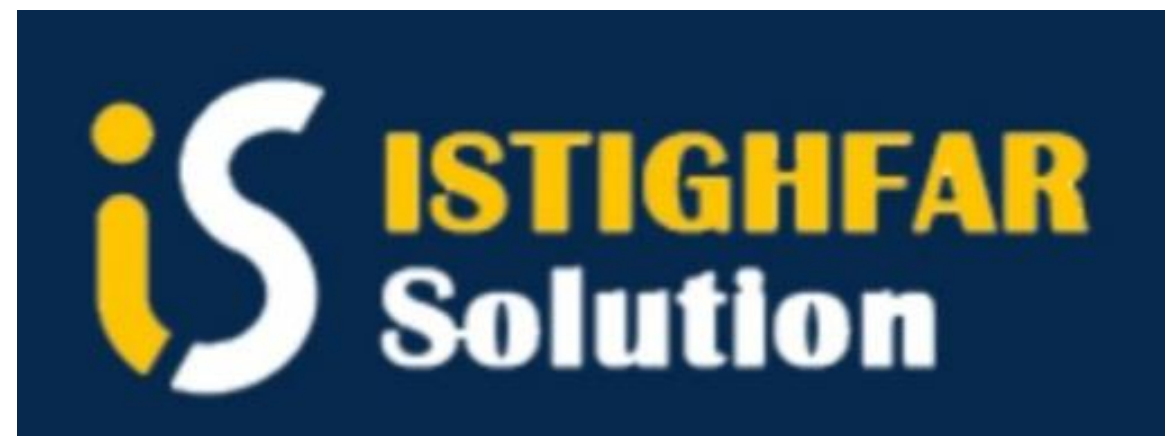

\section{POLIMERISASI}

1. POLIMERISASI ADISI

Contoh, Polietilena, Polipropilena, Polistirena, Teflon, PVC, dan Poliisoprena (Karet Alam).

2. POLIMERISASI KONDENSASI

Contoh, Protein, Poliester, Plastik Bakelit, dan Nilon. 